\title{
Bilateral lower limb paralysis as initial symptom of neurofibromatosis type 2: A case report
}

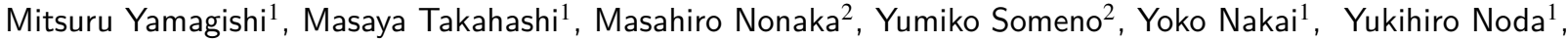 \\ Hirohide Kawasaki*1, Kazunari Kaneko ${ }^{1}$ \\ ${ }^{1}$ Departments of Pediatrics, Kansai Medical University, Hirakata, Osaka, Japan \\ ${ }^{2}$ Departments of Neurosurgery, Kansai Medical University, Hirakata, Osaka, Japan
}

Received: December 10, 2016

Accepted: January 29, 2017

Online Published: February 12, 2017

DOI: $10.5430 /$ ijdi.v4n1p24

URL: http://dx.doi.org/10.5430/ijdi.v4n1p24

\begin{abstract}
Neurofibromatosis type 2 (NF2) is an autosomal dominant disorder characterized by multiple tumors including schwannomas, meningiomas and ependymomas of the central nervous system. Although paresthesias, muscle weakness, muscle atrophy, and gait unsteadiness have been reported as clinical symptoms of spinal tumors, there are no reports of patients with NF2 who developed paralysis. A 7-year-old female consulted with our hospital because of sudden bilateral lower limb paralysis. She had not recognized the abnormalities, including paralysis, paraplegia and muscle weakness of bilateral lower limb before administration. Because of the aggravated bilateral patellar tendon reflexes, Achilles tendon reflexes, and the positive bilateral Babinski reflexes, a magnetic resonance imaging (MRI) of the spinal cord was performed. MRI revealed multiple spinal tumors in the cervical, thoracic, lumbar, and sacral regions. In particular, a $12 \mathrm{~mm} \times 33 \mathrm{~mm}$ spinal tumor was present in the thoracic region and was pressing against the spinal cord. Surgery was urgently performed to remove the spinal tumor in the thoracic region. Histopathological examination revealed that the tumor was a schwannoma. Gadolinium-enhanced MRI of the head was performed to explore the intra-cranial lesions: bilateral vestibular schwannomas and tumors in the left oculomotor nerve and right glossopharyngeal nerve were also discovered. Auditory brainstem response was performed to evaluate the bilateral VSs, and the results were normal. Nine months after the surgery, MRI of the head and spinal cord showed that her tumors had not increased in size. This represents the first reported case of NF2 complicated bilateral lower limb paralysis as initial neurological symptom.
\end{abstract}

Key Words: Neurofibromatosis type 2, Spinal tumor, Paralysis, Magnetic resonance imaging

\section{INTRODUCTION}

Neurofibromatosis type 2 (NF2) is an autosomal dominant disorder characterized by multiple tumors including schwannomas, meningiomas and ependymomas of the central nervous system. NF2 is rare and has a reported incidence of 1:25,000 to $1: 40,000 .^{[1,2]}$ Bilateral vestibular schwannomas (VSs) develop in more than $90 \%$ of patients with NF2, and approximately half of these patients complain of deafness. ${ }^{[3]}$
Other neurologic lesions, including those involving other cranial nerves, the spinal cords and peripheral nerves, are common lesions in patients with NF2. Cataracts and cutaneous tumors are also known as clinical manifestations of NF2.

Most patients with NF2 eventually develop a spinal tumor, usually a schwannoma the clinical manifestations of spinal tumors are debilitating pain, paresthesias, muscle weakness,

${ }^{*}$ Correspondence: Hirohide Kawasaki, MD, PhD; Email: kawasaki@hirakata.kmu.ac.jp; Address: Department of Pediatrics, Kansai Medical University, 2-5-1 Shinmachi, Hirakata, Osaka 573-1010, Japan. 
and muscle atrophy. ${ }^{[3,4]}$ No report has described a child with NF2 who developed sudden bilateral lower limb paralysis.

We herein describe a 7-year-old female with NF2 who developed sudden bilateral lower limb paralysis and discuss the clinical features of the disease.

\section{Case presentation}

A 7-year-old female consulted with our hospital because of sudden bilateral lower limb paralysis. She had a 5-day history of a low-grade fever, cough, and nausea. She had no relevant family history. She had not recognized the abnormalities, including paralysis, paraplegia and muscle weakness of bilateral lower limb before administration. Neurological examination revealed an aggravated bilateral patellar tendon reflexes, and Achilles tendon reflexes, as well as a positive, bilateral Babinski reflexes. Physical examination revealed no abnormalities associated with the lungs, heart, or abdomen. A chest X-ray was also normal. Laboratory findings showed a normal white blood cell count $(5,700 / \mu \mathrm{l})$ and slight increase in the $\mathrm{C}$-reactive protein concentration $(1.0 \mathrm{mg} / \mathrm{dl}$; normal range $<0.3 \mathrm{mg} / \mathrm{dl})$. The patient had no anemia or thrombocytopenia. Her creatinine, aspartate amino transferase, alanine transaminase, and lactate dehydrogenase concentrations were also normal. Because of the aggravated bilateral patellar tendon reflexes, Achilles tendon reflexes, and the positive bilateral Babinski reflexes, a magnetic resonance imaging (MRI) of the spinal cord was performed. MRI revealed multiple spinal tumors in the cervical, thoracic, lumbar, and sacral regions. In particular, a $12 \mathrm{~mm} \times 33 \mathrm{~mm}$ spinal tumor was present in the thoracic region and was pressing against the spinal cord (see Figure 1). Surgery was urgently performed to remove the spinal tumor in the thoracic region. Histopathological examination revealed that the tumor was a schwannoma with atypical nuclei exhibiting high chromatin density (see Figure 2). Based on these results, gadolinium-enhanced MRI of the head was performed to explore the intra-cranial lesions: bilateral VSs and tumors in the left oculomotor nerve and right glossopharyngeal nerve were also discovered (see Figure 3); however, no tumors were discovered in the meninges. One café au lait spot was present on the abdomen. Therefore, the patient was diagnosed with NF2. The day after the surgery, her bilateral patellar tendon reflexes, and Achilles reflexes had normalized. In addition, her bilateral Babinski reflex has turned to be negative. The next day, she was able to walk normally. Auditory brainstem response (ABR) was performed to evaluate the bilateral VSs, and the results were normal. There were no other neurological symptoms, cataracts, or cutaneous tumors.

Published by Sciedu Press
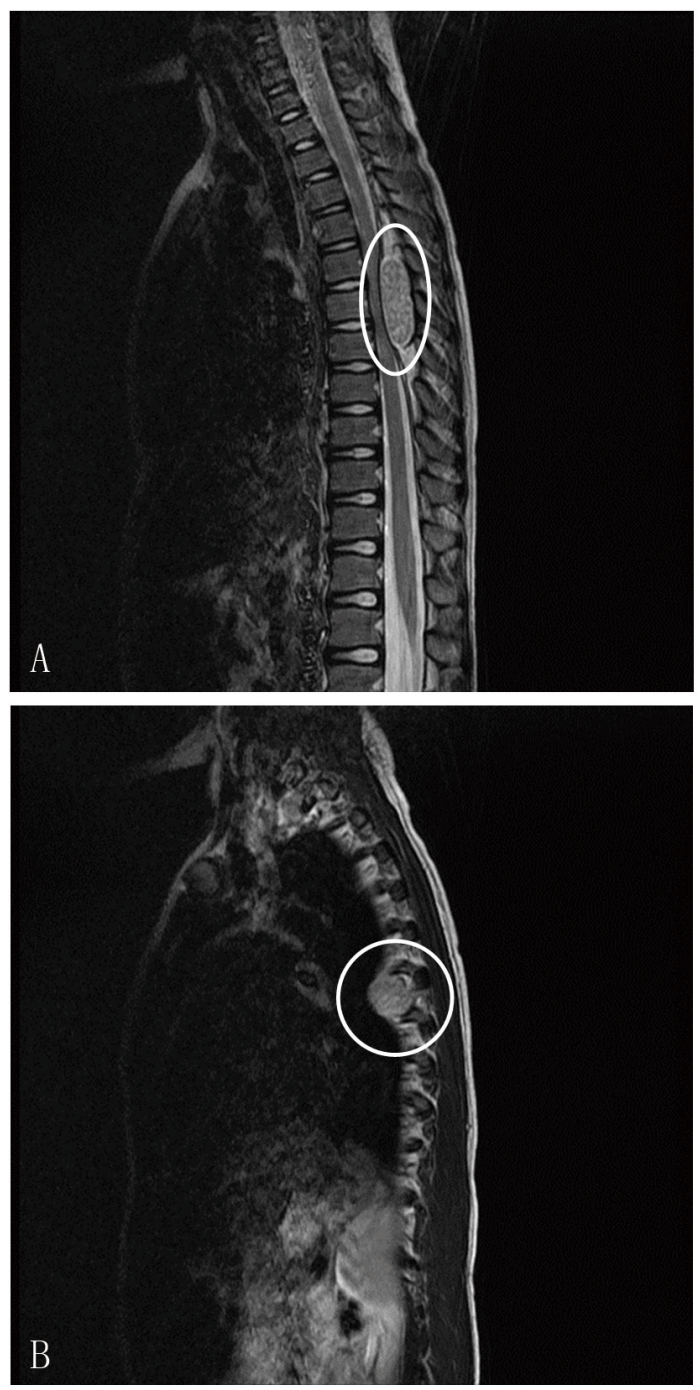

Figure 1. T2-weighted sagittal magnetic resonance image. A tumor of the extradural space is pressing against the spinal cord in the thoracic region (Th5-7). Between Th6 and Th7, the tumor develop an intervertebral foramen and under a thoracic cavity

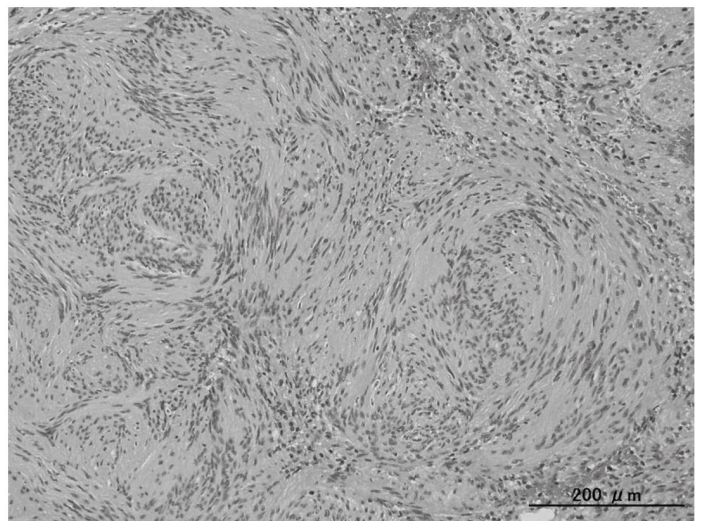

Figure 2. Histopathological findings of the tumor in the thoracic region. The tumor was a schwannoma with atypical nuclei exhibiting high chromatin density 

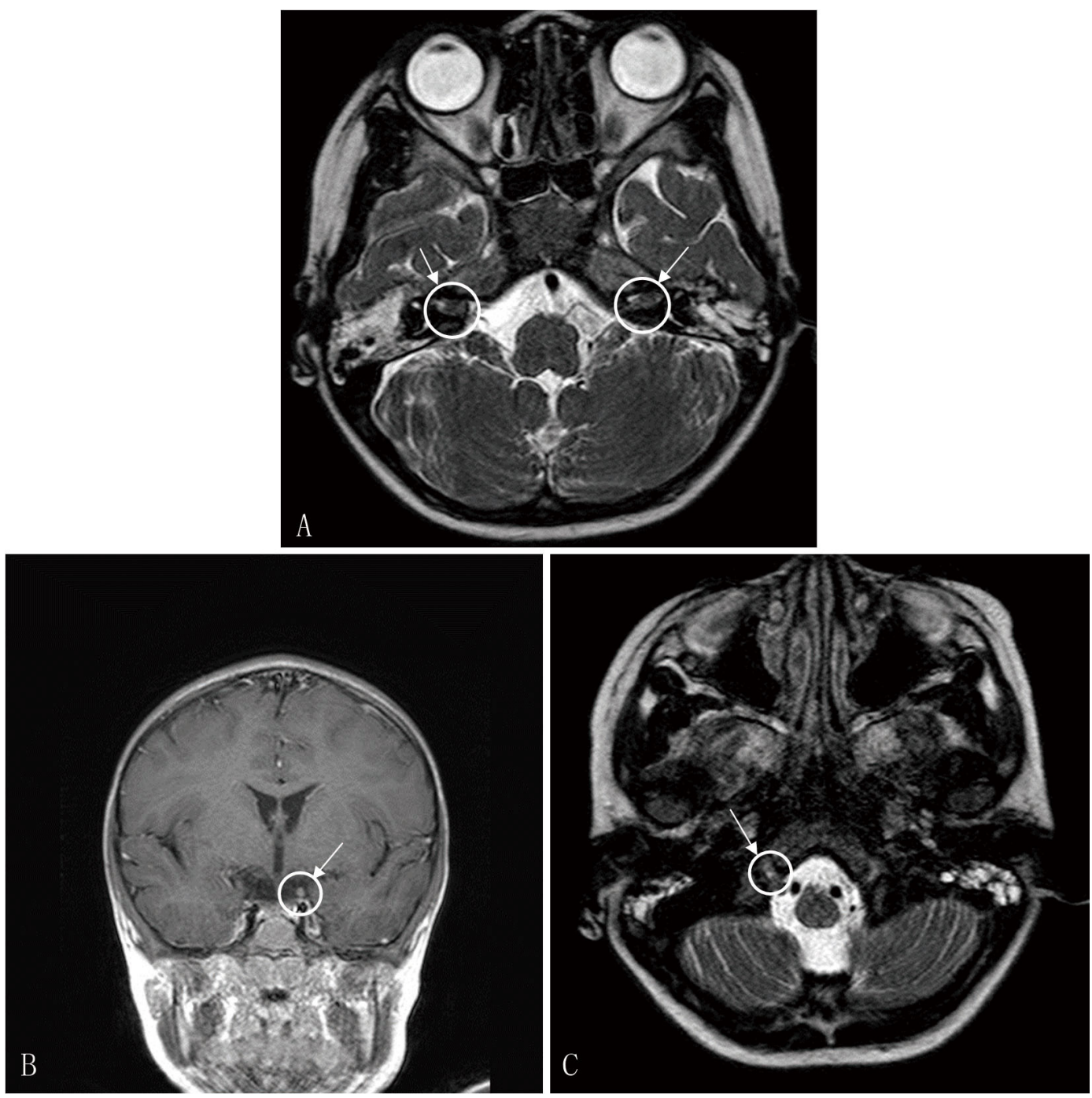

Figure 3. Gadolinium-enhanced MRI of the head. A: Bilateral vestibular schwannomas; B: Tumors in the left oculomotor nerve; $\mathrm{C}$ : Tumors in right glossopharyngeal nerve

Nine months after the surgery, the patient had no neurological symptoms. Her ABR results were normal. MRI of the head and spinal cord showed that her tumors had not increased in size.

\section{Discussion}

We have presented a case involving a 7-year-old female with NF2 who developed bilateral lower limb paralysis. Most cases of NF2 usually manifest clinical features in adulthood. A large-scale, retrospective study by Evans et al. ${ }^{[4]}$ showed that only $18 \%$ of NF2 developed in childhood. Children who develop NF2 may exhibit a seriously worse course because of multiple tumors. ${ }^{[3,5]} \mathrm{NF} 2$ is a dominantly inherited syndrome caused by mutations of the NF2 gene, which is located on chromosome $22,{ }^{[6]}$ while most pediatric cases were sporadic ${ }^{[4]}$ as well in the present case: neither the parents nor grandparents showed clinical manifestations of NF2.
Although her NF2 gene was not investigated, she was diagnosed with NF2 based on the clinical criteria established at the National Institution Health consensus conference. ${ }^{[7]}$

Although NF2 is well known to present with symptoms (hearing loss, tinnitus, and facial palsy) caused by VSs, Evans et al. ${ }^{[4]}$ revealed that less than one-third of all patients presented with VS-related symptoms. However, almost all patients developed a VS 1 to 26 years after the diagnosis of NF2 in their study. ${ }^{[4]}$ Although our patient did not present with symptoms of a VS, gadolinium-enhanced MRI revealed bilateral VSs. ABR was therefore performed, and the results were normal. However, regular ABR should be performed to discover symptomatic VSs earlier.

Spinal tumors are frequently found in patients with NF2. Parry et al. reported that $33(67.4 \%)$ of 49 patients who underwent imaging studies had spinal tumors: ${ }^{[3]}$ however, 
the initial symptoms caused by spinal tumors were recognized only in 13 patients $(21 \%)$. These include radiating pain accompanied by paresthesia, muscle weakness, atrophy, gait unsteadiness, and inturning of the foot. According to the report by Evans et al., ${ }^{[4]}$ spinal tumors were discovered based on a clinical symptom (paresthesia) in only $1(3.3 \%)$ of 30 children with NF2 aged $<10$ years and Mautner et al. ${ }^{[8]}$ reported that spinal neurological symptoms were recognized only in $2(4 \%)$ of 48 patients while spinal tumors were found in $43(90 \%)$ among them. Thus, it is usually difficult to discover spinal tumors based on clinical manifestations and spinal tumors were discovered by screening MRI in most patients with NF2. We could not show the reason why she suddenly recognized her bilateral lower limb paralysis, although the growth rate of schwannoma was slow.
In the present case, bilateral lower limb paralysis caused by a spinal tumor led us to the diagnosis of NF2. To the best of our knowledge, this is the first case of NF2 who developed bilateral lower limb paralysis as an initial neurological symptom.

Based on these findings, we suggest that although our case is very rare, it should be noted that bilateral lower limb paralysis can be the initial symptom of NF2.

\section{ACKNOWLedgements}

This study was supported by the Mami Mizutani Foundation.

\section{CONFlicts OF InTEREST Disclosure}

All authors have declared that they have no conflicts of interest.

\section{REFERENCES}

[1] Evans DG, Moran A, King A, et al. Incidence of vestibular schwannoma and neurofibromatosis 2 in the North West of England over a 10-year period: higher incidence than previously thought. Otol Neurotol. 2005; 26: 93-97. PMid:15699726 https ://doi .org/10 .1097/00129492-200501000-00016

[2] Evans DG, Huson SM, Donnai D, et al. A genetic study of type 2 neurofibromatosis in the United Kingdom. I. Prevalence, mutation rate, fitness, and confirmation of maternal transmission effect on severity. J Med Genet. 1992; 29: 841-846. PMid:1479598 https://doi.org/10.1136/jmg.29.12.841

[3] Parry DM, Eldridge R, Kaiser-Kupfer MI, et al. Neurofibromatosis 2 (NF2): clinical characteristics of 63 affected individuals and clinical evidence for heterogeneity. Am J Med Genet. 1994; 52: 450-461. PMid:7747758 https://doi.org/10.1002/ajmg. 1320520411
[4] Evans DG, Birch JM, Ramsden RT. Paediatric presentation of type 2 neurofibromatosis. Arch Dis Child. 1999; 81: 496-499. PMid:10569966 https://doi.org/10.1136/adc.81.6.496

[5] Evans DG, Huson SM, Donnai D, et al. A clinical study of type 2 neurofibromatosis. Q J Med. 1992; 84: 603-618. PMid:1484939

[6] Seizinger BR, Martuza RL, Gusella JF. Loss of genes on chromosome 22 in tumorigenesis of human acoustic neuroma. Nature. 1986; 322: 644-647. PMid:3092103 https ://doi .org/10.1038/322644a0

[7] Neurofibromatosis. Conference statement. National Institutes of Health Consensus Development Conference. Arch Neurol. 1988; 45: 575-578. PMid:3128965

[8] Mautner VF, Lindenau M, Baser ME, et al. The neuroimaging and clinical spectrum of neurofibromatosis 2 . Neurosurgery. 1996: 38 : 880-885. PMid:8727812 https://doi .org/10.1097/00006123 $-199605000-00004$ 